\title{
Theoretical and Experimental Study of Spectral Selectivity Surface for Both Solar Heating and Radiative Cooling
}

\author{
Mingke Hu, ${ }^{1}$ Gang Pei, ${ }^{1}$ Lei Li, ${ }^{2}$ Renchun Zheng, ${ }^{1}$ Junfei Li, ${ }^{1}$ and Jie $\mathrm{Ji}^{1}$ \\ ${ }^{1}$ Department of Thermal Science and Energy Engineering, University of Science and Technology of China, Hefei 230027, China \\ ${ }^{2}$ Eastern Boiler Control CO., LTD., Shenzhen 518057, China \\ Correspondence should be addressed to Gang Pei; peigang@ustc.edu.cn
}

Received 10 December 2014; Revised 2 June 2015; Accepted 3 June 2015

Academic Editor: Mark van Der Auweraer

Copyright (c) 2015 Mingke Hu et al. This is an open access article distributed under the Creative Commons Attribution License, which permits unrestricted use, distribution, and reproduction in any medium, provided the original work is properly cited.

\begin{abstract}
A spectral selectivity surface for both solar heating and radiative cooling was proposed. It has a high spectral absorptivity (emissivity) in the solar radiation band and atmospheric window band (i.e., $0.2 \sim 3 \mu \mathrm{m}$ and $8 \sim 13 \mu \mathrm{m}$ ), as well as a low absorptivity (emissivity) in other bands aside from the solar radiation and atmospheric window wavelengths (i.e., $3 \sim 8 \mu \mathrm{m}$ or above $13 \mu \mathrm{m}$ ). A type of composite surface sample was trial-manufactured combining titanium-based solar selective absorbing coating with polyethylene terephthalate (TPET). Sample tests showed that the TPET composite surface has clear spectral selectivity in the spectra of solar heating and radiation cooling wavelengths. The equilibrium temperatures of the TPET surface under different sky conditions or different inclination angles of surface were tested at both day and night. Numerical analysis and comparisons among the TPET composite surface and three other typical surfaces were also performed. These comparisons indicated that the TPET composite surface had a relative heat efficiency of $76.8 \%$ of that of the conventional solar heating surface and a relative temperature difference of $75.0 \%$ of that of the conventional radiative cooling surface, with little difference in cooling power.
\end{abstract}

\section{Introduction}

The temperature in outer space is close to absolute zero. In addition, the higher atmosphere is much colder than the surface of the earth. Therefore, the sky is a huge cold storage for objects on the ground. Given the existence of water vapor $\left(\mathrm{H}_{2} \mathrm{O}\right)$, carbon dioxide $\left(\mathrm{CO}_{2}\right)$, and ozone $\left(\mathrm{O}_{3}\right)$, the atmosphere has different transmittances in different wavelengths. In the vast majority of bands, the atmosphere weakens the heat radiation from the ground to outer space. However, in wavelengths such as $0.3 \sim 3,3.2 \sim 4.8$, and 8 $13 \mu \mathrm{m}$, the atmosphere has extremely high transmittance. The band that interests us most is $8 \sim 13 \mu \mathrm{m}$ (i.e., the atmospheric window), wherein the heat radiation of bodies on the ground are mainly concentrated. Objects on the surface of the earth obtain a cooling power by radiating heat to the cold outer space through the atmospheric window. This phenomenon is "radiative cooling."

Radiative cooling has been studied as early as the 1970s. Michell and Biggs [1] carried out temperature measurements in two identical houses roofed by $\mathrm{TiO}_{2}$-pigmented paint applied on metal substrate and aluminized Tedlar roofs. They established that the temperature drop was too small, so the spectral selectivity of the aluminized Tedlar roof could not be exploited fully. Catalanotti et al. [2] realized a selective surface with optical properties that matched the atmospheric window. With respect to a black radiator, this surface is considerably more effective for cooling by being exposed to the clear sky. Erell and Etzion [3] found that keeping the radiating surface relatively warm was the key to increasing radiative cooling power. Therefore, the system they built made up of a roof pond was insulated from the environment. The surface of the flat plate was exposed to the sky through which the water from the pond was cooled through convection and radiation. In their another research [4], they analyzed the parameters that affect the performance of a flat-plate radiator designed for radiative cooling. An affordable, simple, and flexible design for a cooling radiator was suggested as a result of the analysis. The design was tested at the experimental facilities of the Center for Desert Architecture at Sde Boker, Israel. The mean nightly cooling output of the radiator (due to the combined effect of radiation and convection) was over 
$90 \mathrm{~W} / \mathrm{m}^{2}$ under typical desert meteorological conditions. Via optimised combinations of aperture geometry and spectral emittance profiles of surfaces and the atmosphere, Smith [5] found that an amplified radiative cooling power up to $135 \mathrm{~W} / \mathrm{m}^{2}$ could be achieved. Zhang and Niu [6] proposed a novel microencapsulated phase change material (MPCM) slurry storage system by combining MPCM slurry storage with a nocturnal radiative cooling system. The cooling energy consumption and the effect of energy-free radiation application were simulated in five typical cities across China. Bagiorgas and Mihalakakou [7] established an aluminum nocturnal radiator painted with appropriate white paint. The dynamic thermal performance of the system during summer was calculated using an accurate mathematical model through an extensive validation process. FarmahiniFarahani and Heidarinejad [8] established a multistep system of nocturnal radiative cooling and two-stage evaporative cooling, in which the nocturnal radiative cooling is used as a precooling process to increase the subsequent evaporative cooling step. The feasibility and potential of this system was investigated for four cities which have different climate. Meir et al. [9] presented a radiative cooling system that consisted of unglazed flat-plate radiators, water as heat carrier, and a reservoir. In addition, the effect of tilt angle, aperture area, and reservoir volume on cooling performance has been studied in simulations. Ge and Sun [10] summarized the principle of radiative cooling and established the radiative heat transfer model between the sky and the radiator. The authors also conducted a sample calculation and analysis. $\mathrm{Li}$ and Jiang [11] selected a set of compounds that can function as radiators through the Sadtler standard spectra and studied the fabrication process of radiating surface. Coatings and tiles such as $\mathrm{SiO}, \mathrm{MgO}$, and $\mathrm{LiF}$ have been tested, yielding results that suggest the possibility of radiative cooling at night $[12$, 13]. Rui and Zuo [14] conducted experimental tests on several radiative materials. The results showed that the equilibrium temperature of polyethylene terephthalate film (PET) was $11^{\circ} \mathrm{C}$ lower than the ambient temperature under clear sky conditions. Saitoh and Fujino [15] indicated that sky radiation cooling appears most significant under certain weather conditions, namely, clear weather with low relative humidity and low wind speeds. Polyethylene is the most commonly used convection cover to date, despite its vulnerability to rapid degradation by solar ultraviolet. Many researchers focused on other durable materials. Zinc sulphide has been found to be a quite durable and suitable material which performs thermally close to polyethylene by Bathgate and Bosi [16]. But they also suggest that a much cheaper way for manufacturing large zinc sulphide tiles needs to be developed in the future. Gentle et al. [17] proposed polyethylene mesh as the convection cover. They found it performed a better performance in durability and limits on unit areas comparing to polyethylene foil and an advantage in transmittance and cost comparing to $\mathrm{ZnS}$ tiles.

Radiative cooling devices are always very simple and can obtain cooling powers without external energy input. However, their cooling powers are not sufficiently large. Moreover, a radiative cooling device cannot act as a flat-plate solar collector during daytime because the radiator has very low spectral absorptivity in solar radiation wavelength (i.e., $0.2 \sim 3 \mu \mathrm{m})$. Meanwhile, a flat-plate solar collector cannot act as a nocturnal cooling radiator because of its extremely low emissivity in the atmospheric window wavelength (i.e., 8 $13 \mu \mathrm{m})$. In several decades, there has been little work reported on solar heating and nocturnal cooling using the same surface except for the original research by Matsuta et al. [18]. They presented a kind of collective-radiative surface made of a black copper plate, coated with a thin polyvinyl-difluoride film which can act as a solar collector at daytime and sky radiator at night. In this paper, we proposed a new spectral selectivity surface for both solar heating during daytime and radiative cooling during nighttime.

Firstly, it is necessary here to talk about the spectral properties of spectral selectivity surface for both solar heating and radiative cooling.

As we all know, the efficiency of a solar collector depends on two major factors: spectral absorptivity in solar radiation wavelength and heating loss. To improve heat efficiency, high solar absorptivity and low heating loss must be ensured. Approximately $99 \%$ of solar radiation is in the $0.2 \sim 3 \mu \mathrm{m}$ range. However, the vast majority of radiation from objects on the earth is in $3 \sim 25 \mu \mathrm{m}$ range. Therefore, the collector surface should possess high spectral absorptivity in the $0.2 \sim$ $3 \mu \mathrm{m}$ range and low spectral emissivity in $3 \sim 25 \mu \mathrm{m}$ range. Solar selective absorbing coatings meet the above requirements and, thus, have been widely used on solar collectors. Their spectral absorptivity (i.e., spectral emissivity) is always higher than 0.9 in solar radiation wavelength and as low as 0.05 in other bands. The cooling effect of radiative cooling devices is also closely related to the spectral properties of the surface. Heat radiation of objects on the ground is mainly concentrated in the atmospheric window wavelength $(8 \sim 13 \mu \mathrm{m})$. Therefore, the radiative cooling surface should have high spectral emissivity in the atmospheric window wavelength, indicating that it could obtain a large cooling power by radiating heat to the cold outer space. Meanwhile, surface temperature is lower than those of the sky and the environment because of heat exchange with the cold outer space and good insulation measures. Thus, the radiative cooling surface should have low spectral absorptivity in those bands excluding atmospheric window wavelength, which indicates that it could reject most radiated heat from the sky and the environment.

Based on the analysis above, to carry out solar heating during daytime and radiative cooling during nighttime, the key is to obtain a spectral selectivity surface that could achieve the two functions. This kind of surface should have high spectral absorptivity (emissivity) in solar radiation and atmospheric window wavelengths so that it could obtain a large heating power during daytime and a large cooling power at nighttime. In addition, the surface should have low spectral absorptivity (emissivity) in other bands aside from the solar radiation and atmospheric window wavelength, allowing it to decrease radiant heating loss during daytime and radiant cooling loss during nighttime. Obviously, the ideal surface for both solar heating and radiative cooling should have 


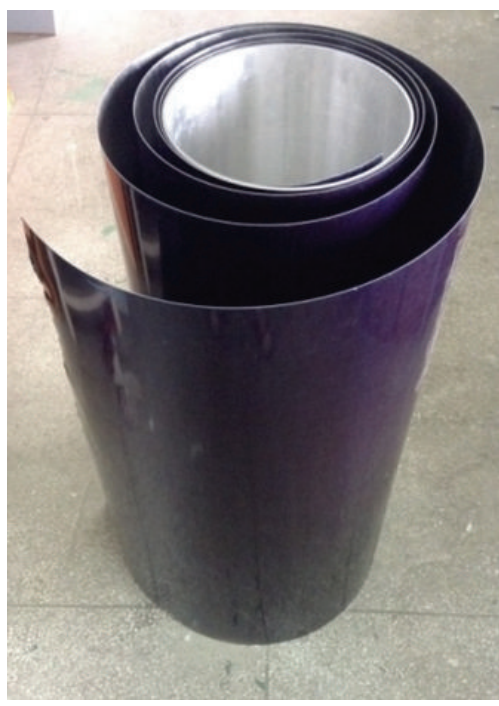

(a) Titanium-based coating

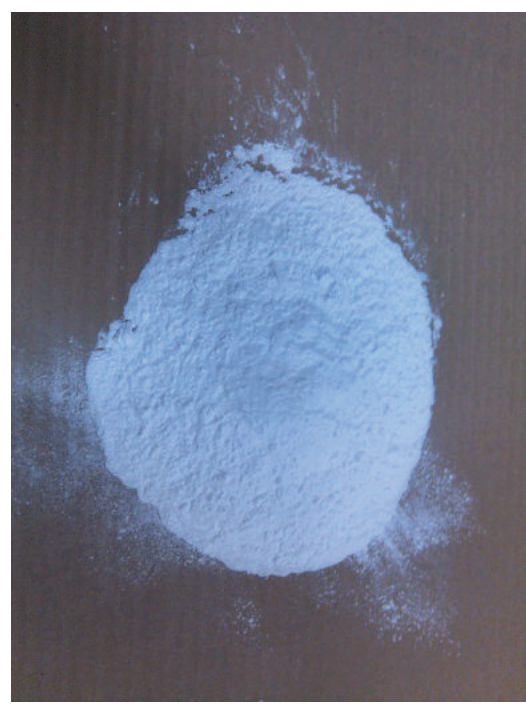

(b) PET powder

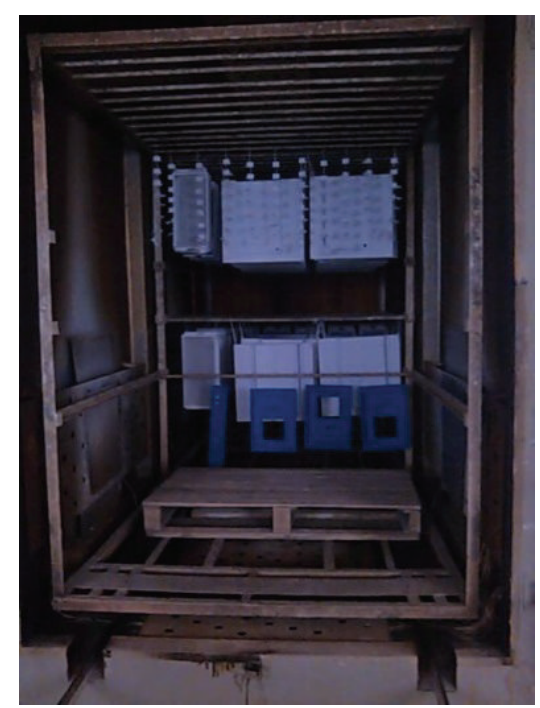

(c) Constant temperature oven

FIGURE 1: Materials and processing facility of the TPET surface.

an absorptivity (emissivity) of $100 \%$ in solar radiation and atmospheric window wavelengths but zero in other bands.

In this study, we trial-manufactured a type of composite surface sample combining titanium-based solar selective absorbing coating with polyethylene terephthalate (TPET). Then the spectral properties of the composite surface were measured. The solar heating and radiative cooling performance of TPET were also compared with three other typical surfaces via numerical analysis. In addition, experimental tests were carried out. A system based on this kind of surface can not only act as a solar heating system in the daytime but also act as a radiative cooling system in the nighttime, which means it could work all through the day to address the limitations of traditional radiative cooling devices and flatplate solar collectors mentioned above and shorten the initial payback period. This TPET composite surface also shows its good potential of practical use in industry since its processing method and cost are simple and cheap.

\section{Trial-Manufacturing, Spectral Measurement and Analysis, and Preliminary Experimental Test}

2.1. Trial-Manufacturing. Solar selective absorbing coatings have spectral properties close to those of an ideal solar collector surface. However, polyethylene terephthalate (PET) film has high spectral emissivity in the atmospheric window band and high spectral transmittance in other bands. Therefore, we propose a composite surface consisting of a solar selective absorbing coating upon which a sheet of PET film is coated. Based on the spectral analysis result, the spectral properties of the composite surface are close to those of the ideal surface for both solar heating and radiative cooling.

In this study, we selected the titanium-based coating (Figure 1(a)) as the solar selective absorbing coating. At the beginning, we cleaned the surface of the titanium-based coating and made it as flat as possible. Then we covered a layer of $30 \mu \mathrm{m}$ thick PET powder (Figure 1(b)) on the titaniumbased coating with a powder monitor. At the next stage, it was sent to a constant temperature $\left(200^{\circ} \mathrm{C}\right)$ oven (Figure 1(c)) for approximately $10 \mathrm{~min}$. The PET powder was turned into film and then attached to the titanium-based coating. The new composite surface after natural cooling was called the TPET surface (Figure 2(b)).

The thickness of the PET film is a key factor of the spectral properties of the composite surface. The thinner the thickness of the PET film is, the closer the spectral properties of the TPET composite surface will be to the ideal surface for both solar heating and radiative cooling. In this paper, the thickness of the PET film is $30 \mu \mathrm{m}$, which is not thin enough compared to that of some other researches $[2,18]$. In further studies, we will work on better ways such as finding more specialized equipment or technologies to lower the thickness of the PET film.

2.2. Spectral Measurement and Analysis. The TPET surface is a radiopaque substance, so its spectral transmittance is zero. Consequently, the spectral absorptivity (emissivity) of the TPET surface can be obtained by testing its spectral reflectivity based on

$$
\alpha_{\lambda}+\rho_{\lambda}=1,
$$

where $\alpha_{\lambda}$ is spectral absorptivity (emissivity) and $\rho_{\lambda}$ is spectral reflectivity. The spectral testing instruments in this study were the UV-Vis-NIR spectrophotometer (DUV-3700) (Figure 3(a)) and Fourier transform infrared spectrophotometer (Bruker Equinox 55) (Figure 3(b)). Both instruments can test spectral reflectivity and transmittance as a function of the wavelength $\lambda$, with spectral regions at $0.2 \sim 2.5 \mu \mathrm{m}$ and 2.5 25 $\mu \mathrm{m}$, respectively. The spectral reflectivity of titaniumbased solar selectively absorbing coating was also tested as 


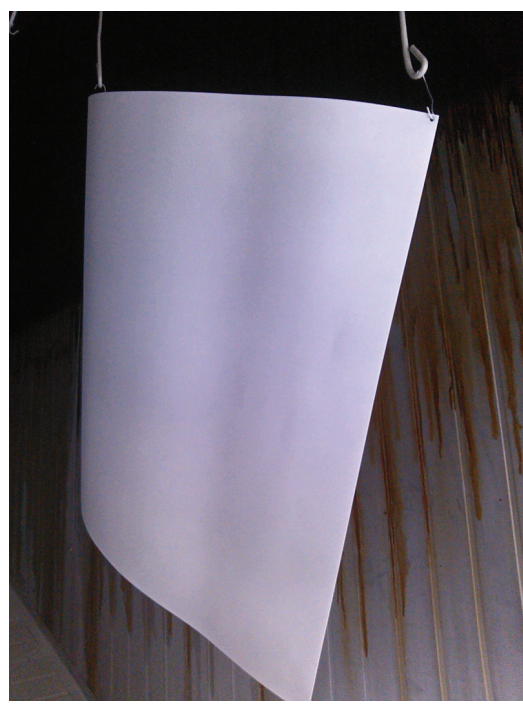

(a)

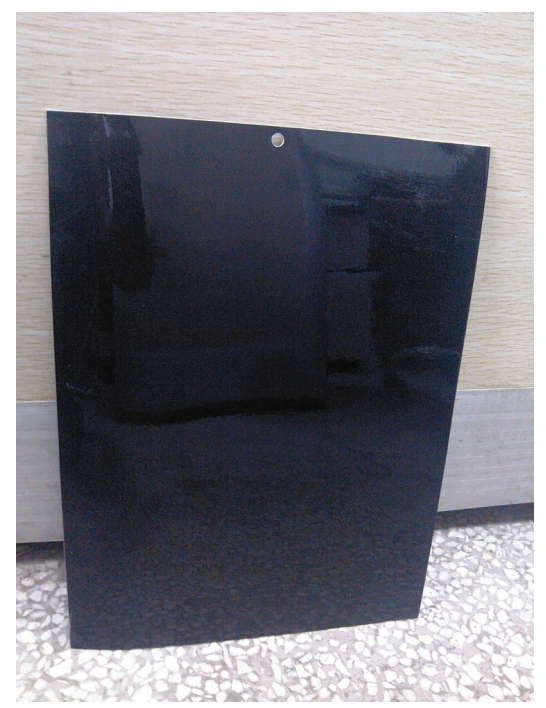

(b)

FIGURE 2: (a) The titanium-based coating surface which was covered by a layer of $30 \mu \mathrm{m}$ thick PET powder and before being sent to a constant temperature $\left(200^{\circ} \mathrm{C}\right)$ oven. (b) The TPET surface.

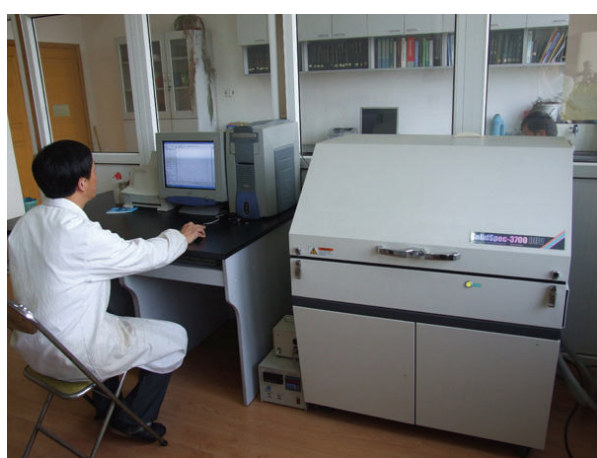

(a) UV-Vis-NIR spectrophotometer (DUV-3700)

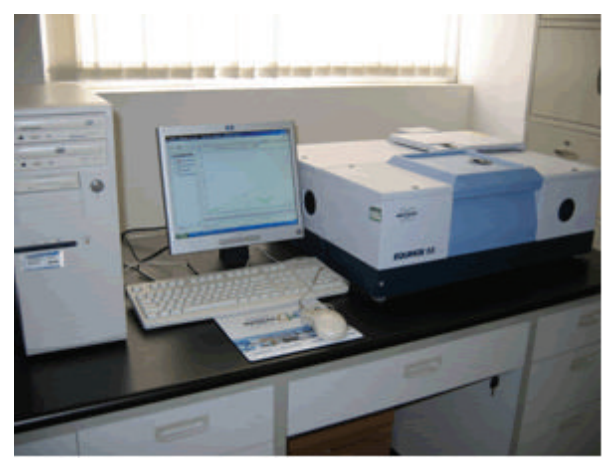

(b) Fourier transform infrared spectrophotometer (Bruker Equinox 55)

FIGURE 3: The spectral testing instruments.

well as the TPET surface in this paper. We tested each sample of these two surfaces three times under the same conditions and then took the average to eradicate any discrepancies.

The results are shown in Figures 4(a) and 4(c). Figure 4(c) shows that the TPET surface has high spectral absorptivity of approximately 0.92 in the solar radiation band, which enables it to absorb the vast majority of solar radiation. The TPET surface has high emissivity of approximately 0.8 in the atmospheric window spectrum, which indicates that it could obtain a large cooling power by radiating heat to the cold outer space. In other bands aside from the solar radiation and atmospheric window wavelengths (i.e., $3 \sim 8 \mu \mathrm{m}$ or above $13 \mu \mathrm{m}$ ), the TPET surface has relatively low absorptivity and emissivity of approximately 0.55 , which allows it to decrease radiant heating loss during daytime and radiant cooling loss during nighttime. Catalanotti et al. [2] tested the spectral reflectivity of a conventional radiative cooling surface (i.e., polyvinyl-fluoride film coating with a sheet of evaporated aluminum). Figure 4(b) shows its spectral absorptivity (emissivity) based on a combination of their experimental results and (1).

2.3. Preliminary Experimental Test. To further analyze the performance qualitatively, especially the radiative cooling effect of the TPET composite surface, experiments were carried out by testing its equilibrium temperatures under different sky conditions or different surface inclination angles. The testing was performed on the night of May 29 and 30, 2014, in Hefei, China. Two different typical weather conditions, namely, clear and cloudy, were presented during the testing. We set four different inclination angles: $0^{\circ}, 30^{\circ}$, $60^{\circ}$, and $90^{\circ}$.

The schematic diagram of the testing system, namely, solar heating and radiative cooling combined system, consisting of a surface for both solar heating and radiative cooling, insulation layer, and windscreen, is shown in Figure 5. Insulation measures are indispensable to ensure that the system obtains considerable heating and cooling efficiencies. 


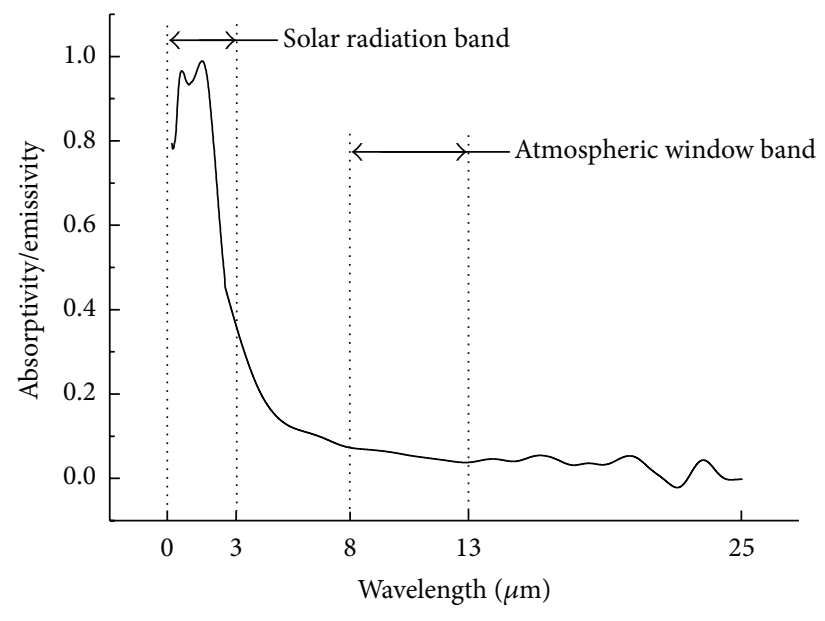

(a) Conventional solar heating surface

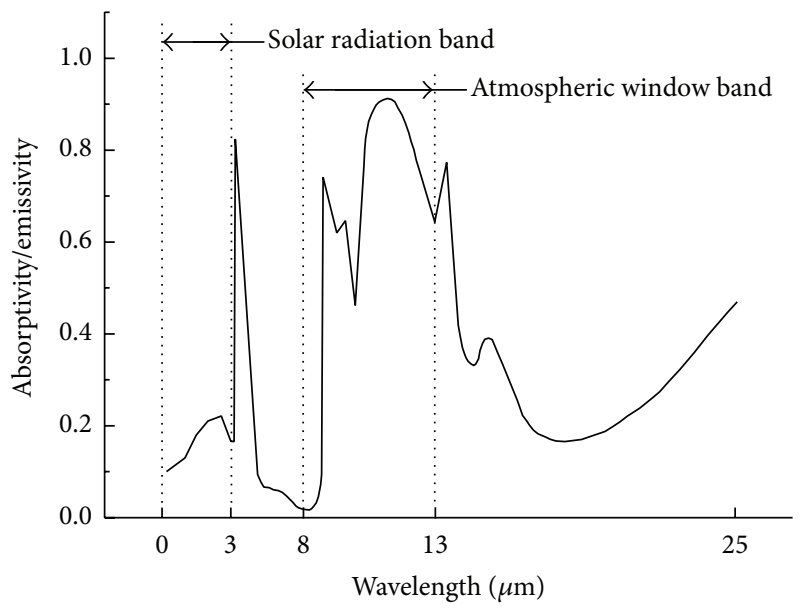

(b) Conventional radiative cooling surface

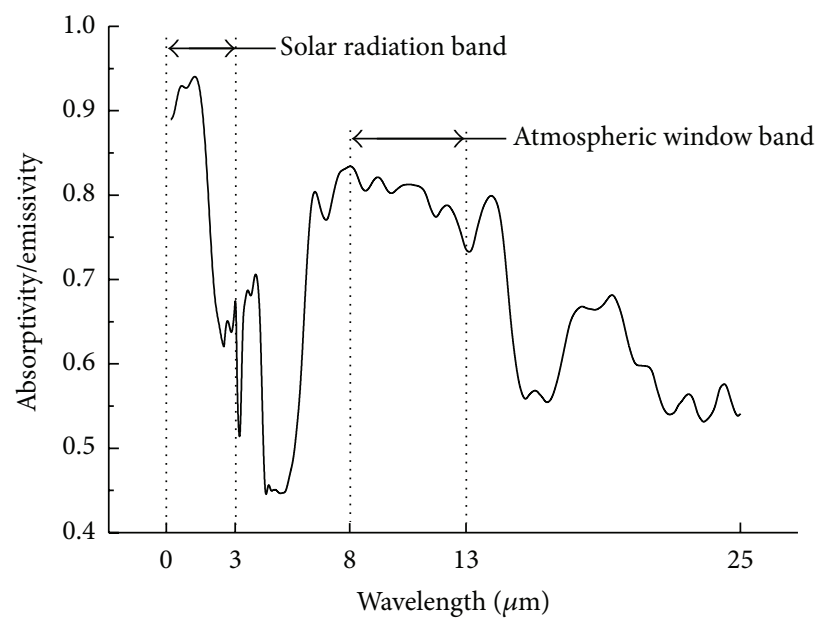

(c) TPET composite surface

FIGURE 4: Spectral absorptivity (emissivity) of three kinds of real surfaces.

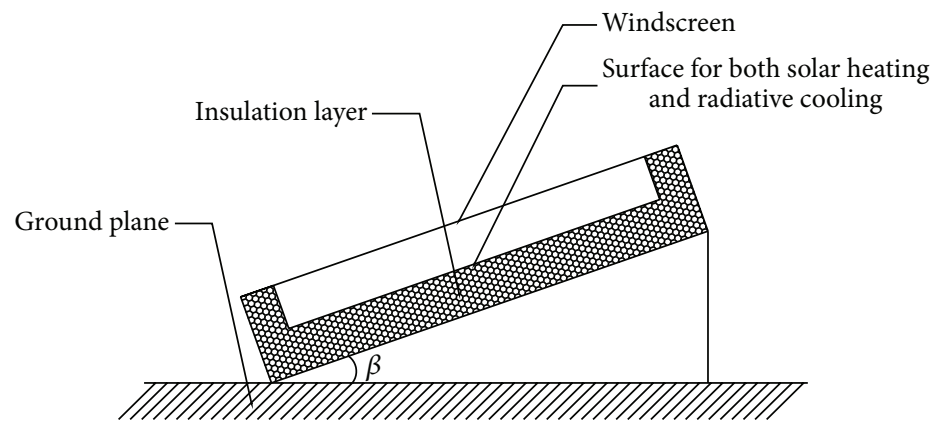

(a) Schematic diagram

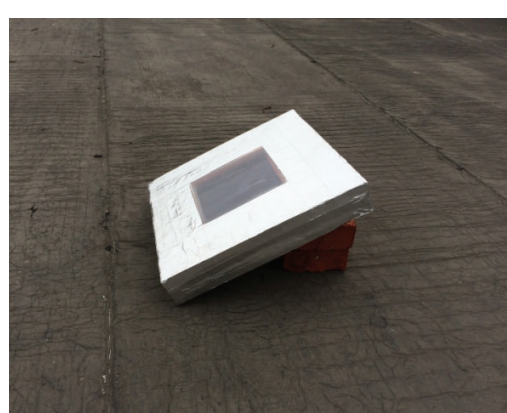

(b) Physical picture

FIGURE 5: Schematic diagram and physical picture of the solar heating and radiative cooling combined system, where $\beta$ is the inclination angle.

Therefore, setting the insulating layer at the bottom and around the surface to decrease heat conduction is necessary. It must also be covered by a windscreen at the top to reduce heat convection. The insulation layer thickness in our system is $50 \mathrm{~mm}$. The radiating area is $200 * 200 \mathrm{~mm}$, and the interval between the radiator and windscreen is $20 \mathrm{~mm}$. Notably, the windscreen should have high spectral transmittance in solar radiation and atmospheric window wavelengths, which enables most radiation in the two regions to go through it. Therefore, the glazing cover used in a solar 


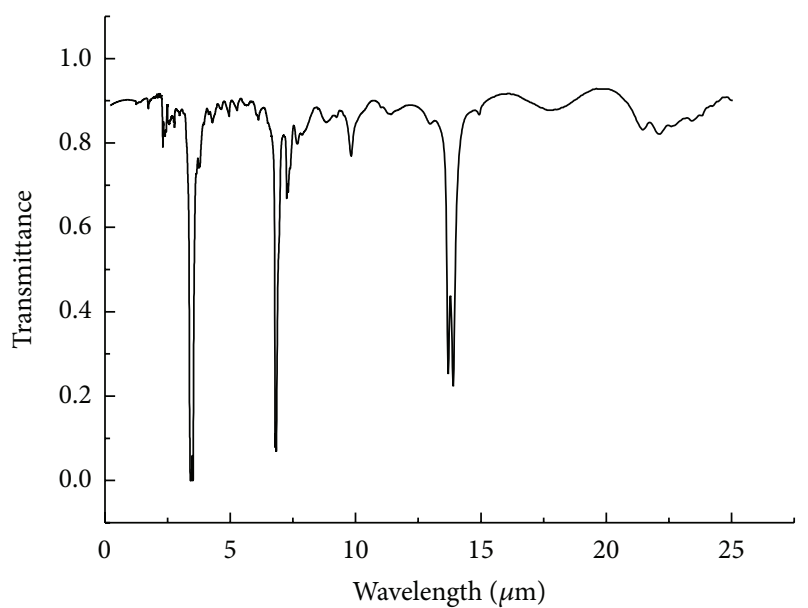

FIGURE 6: Spectral transmittance of low-density polyethylene film.

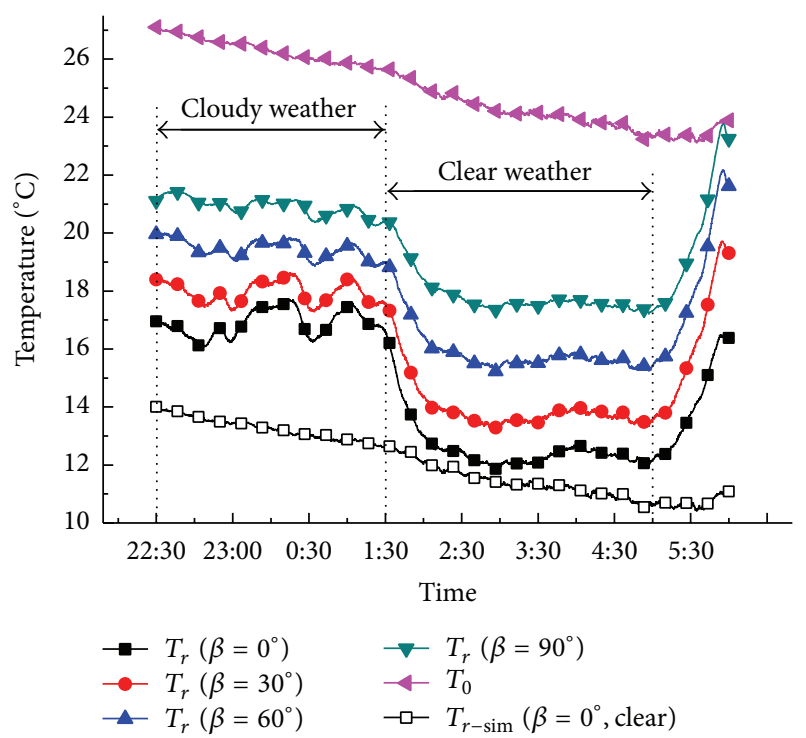

Figure 7: Testing results from 22:30, May 29, to 6:00, May 30, 2014.

collector cannot act as a windscreen due to its low spectral transmittance in the infrared band. Polyethylene film has a high spectral transmittance in the entire $0.2 \sim 25 \mu \mathrm{m}$ region, which indicates that it is a favorable windscreen for both solar heating and radiative cooling. In this study, we chose lowdensity polyethylene film ( $20 \mu \mathrm{m}$ thick) as the windscreen and tested its spectral transmittance in $0.2 \sim 25 \mu \mathrm{m}$ by the UV-Vis-NIR spectrophotometer (DUV-3700) and Fourier transform infrared spectrophotometer (Bruker Equinox 55). The testing result is shown in Figure 6.

The testing results are presented in Figure 7. During the testing period, the ambient temperature $T_{0}$ falls from about $27^{\circ} \mathrm{C}$ in the evening to below $24^{\circ} \mathrm{C}$ just before sunrise. The weather was cloudy from 22:30 to 1:30 while it became clear after 1:30 on the testing day. Thus, the equilibrium temperature of each surface presented sharp drops of $3^{\circ} \mathrm{C}$ to $5^{\circ} \mathrm{C}$ at approximately 1:30. Thus, the radiative cooling effect is better on clear days than on cloudy weather. After 5:00,

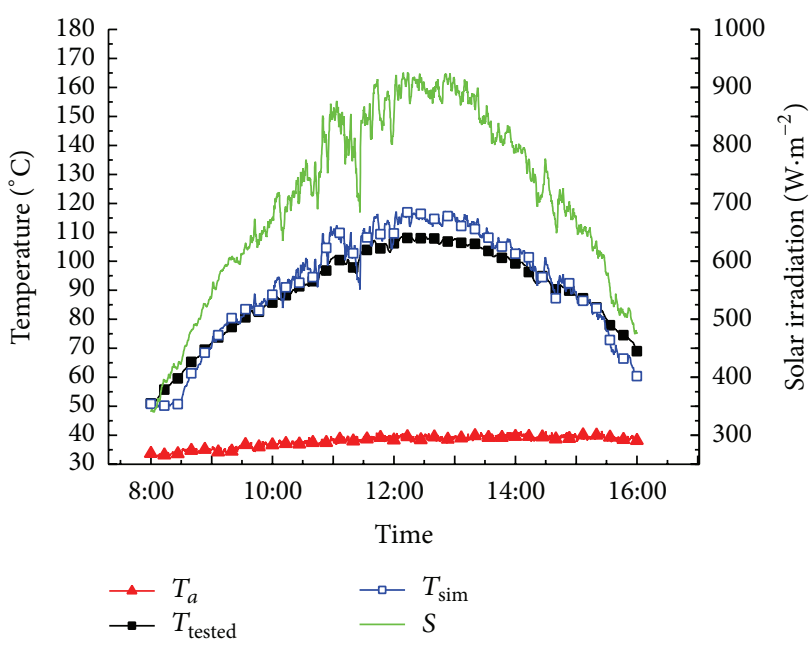

FIGURE 8: Testing results on July 22, 2014, where $T_{a}, T_{\text {tested }}, T_{\text {sim }}$ are the ambient temperature and tested and simulated temperature of the TPET surface, respectively, and $S$ is the solar irradiation.

the temperature of the TPET composite surface started to increase because of the sunrise. However, the equilibrium temperature increased when the inclination angle changed from $0^{\circ}$ to $90^{\circ}$, indicating that the cooling effect worsened as the inclination angle increased. Moreover, under the most favorable condition (i.e., clear sky and inclination angle of $0^{\circ}$ ), the equilibrium temperature of the TPET composite surface was about $13^{\circ} \mathrm{C}$ lower than ambient temperature.

In addition, the equilibrium temperatures of the TPET surface during the day were tested on July 22, 2014, to analyze its solar heating performance. We chose an inclination angle of $32^{\circ}$ because it was the optimal situation for solar heating in Hefei, China $\left(31^{\circ} 51^{\prime} \mathrm{N}, 117^{\circ} 17^{\prime} \mathrm{E}\right)$. The results are presented in Figure 8. It was sunny during the day, and the ambient temperature was always higher than $30^{\circ} \mathrm{C}$ with minimal fluctuations. The equilibrium temperatures of the TPET surface increased from 8:00 to 12:30 and then fell from 12:30 to 16:00, showing a trend similar to that of solar irradiation. The average and maximum values of solar irradiation of the TPET surface were 710 and $925 \mathrm{~W} / \mathrm{m}^{2}$, respectively. Moreover, the average and maximum values of the equilibrium temperature of the TPET surface were $90.4^{\circ} \mathrm{C}$ and $109.7^{\circ} \mathrm{C}$, respectively. Thus, the TPET surface demonstrated considerable solar heating capacity.

\section{Theoretical Analysis}

(a) Solar energy absorbed by the combined system $-q_{1}$ :

$$
q_{1}=\tau_{c} \cdot \alpha_{r} \cdot S,
$$

where $\tau_{c}$ is the transmittance of the windscreen in the solar radiation band, $\alpha_{r}$ is the absorptivity of the surface for both solar heating and radiative cooling, and $S$ is the solar irradiation $\left(\mathrm{W} / \mathrm{m}^{2}\right)$. 
(b) Radiation absorbed from the sky to the combined system $-q_{2}$ :

$$
\begin{aligned}
q_{2} & =2 \int_{0}^{\infty} \int_{0}^{\pi / 2} \varepsilon_{s, \lambda}(\lambda, \theta) \cdot E_{b, \lambda}\left(\lambda, T_{0}\right) \cdot \alpha_{r, \lambda}(\lambda, \theta) \\
& \cdot \tau_{c, \lambda}(\lambda, \theta) \sin \theta \cos \theta d \theta d \lambda
\end{aligned}
$$

where $T_{0}$ is the ambient temperature $(\mathrm{K}), \theta$ is the zenith angle, $\varepsilon_{s, \lambda}(\lambda, \theta)$ is the directional monochromatic emissivity of the atmosphere, $E_{b, \lambda}\left(\lambda, T_{0}\right)$ is the monochromatic radiation intensity of the blackbody under ambient temperature $\left(\mathrm{W} /\left(\mathrm{m}^{2} \cdot \mu \mathrm{m}\right)\right), \alpha_{r, \lambda}(\lambda, \theta)$ is the directional monochromatic absorptivity of the surface for both solar heating and radiative cooling, and $\tau_{c, \lambda}(\lambda, \theta)$ is the directional monochromatic transmittance of the windscreen.

(c) Radiation loss of the combined system $-q_{3}$ :

$$
q_{3}=\int_{0}^{\infty}\left[\frac{E_{b, \lambda}\left(T_{r}\right) \cdot\left(1-\rho_{c, \lambda}\right)-\varepsilon_{c, \lambda} \cdot E_{b, \lambda}\left(T_{c}\right)}{1 / \varepsilon_{r, \lambda}-\left(\left(1-\varepsilon_{r, \lambda}\right) / \varepsilon_{r, \lambda}\right) \cdot \rho_{c, \lambda}}\right] d \lambda,
$$

where $T_{r}$ and $T_{c}$ are the temperature of the surface and windscreen, respectively. $(\mathrm{K})$ and $T_{c}$ are always close to $T_{0}$ in practical situations. $E_{b, \lambda}\left(T_{r}\right)$ and $E_{b, \lambda}\left(T_{c}\right)$ are the monochromatic radiation intensities under $T_{r}$ and $T_{c}$, respectively $\left(\mathrm{W} /\left(\mathrm{m}^{2} \cdot \mu \mathrm{m}\right)\right) ; \rho_{c, \lambda}$ is the monochromatic reflectivity of the windscreen; and $\varepsilon_{r, \lambda}$ and $\varepsilon_{c, \lambda}$ are the monochromatic emissivities of the surface and windscreen.

(d) Heat exchange between the combined system and environment $-q_{4}$ :

$$
q_{4}=\frac{\left|T_{r}-T_{0}\right|}{R_{r a}}
$$

where $R_{r a}$ is the total thermal resistance between the combined system and environment:

$$
R_{r a}=\frac{d_{\mathrm{ins}}}{k_{\mathrm{ins}}}+\frac{1}{h_{a}}
$$

where $d_{\text {ins }}$ is the thickness of the insulation $(\mathrm{m}), k_{\text {ins }}$ is the thermal conductivity of the insulation $(\mathrm{W} /(\mathrm{m} \cdot \mathrm{K}))$, and $h_{a}$ is the convective heat transfer coefficient between the combined system and environment $\left(\mathrm{W} /\left(\mathrm{m}^{2} \cdot \mathrm{K}\right)\right)$. For a radiator covered by a single polyethylene film windscreen, $h_{a}=0.5+1.2 u^{0.5}$ [19], where $u$ is the wind speed $(\mathrm{m} / \mathrm{s})$.

(e) Heat gain of the combined system in solar heating model $-q_{5}$ :

$$
q_{5}=q_{1}+q_{2}-q_{3}-q_{4}
$$

(f) Cooling power of the combined system in radiative cooling model $-q_{6}$ :

$$
q_{6}=q_{3}-q_{2}-q_{4}
$$

(g) $\eta_{1}$ is the relative heating efficiency of the combined system relative to the traditional flat-plate solar collector:

$$
\eta_{1}=\frac{q_{5}}{q_{1}^{\prime}} \times 100 \%
$$

where $q_{1}^{\prime}$ is the heat gain of the traditional flat-plate solar collector $\left(\mathrm{W} / \mathrm{m}^{2}\right)$.

(h) $\eta_{2}$ is the relative temperature difference of the composite surface relative to the conventional radiative cooling surface:

$$
\eta_{2}=\frac{T_{0}-T_{r}}{T_{0}-T_{r}^{\prime}} \times 100 \%
$$

where $T_{r}^{\prime}$ is the equilibrium temperature of the conventional radiative cooling surface $(\mathrm{K})$.

\section{Results and Discussion}

In the radiative cooling mode, the equilibrium temperature of the TPET composite surface with the change in ambient temperature tested from 22:30, May 29, 2014, to 6:00, May 30, 2014, was calculated. The inclination angle of the TPET composite surface was set as $0^{\circ}$. The numerical results are shown in Figure 7. Compared with the results of the equilibrium temperature under inclination angle of $0^{\circ}$, an accepted agreement under clear weather was observed. A difference of approximately $3^{\circ} \mathrm{C}$ was observed under cloudy weather. In the solar heating model, the inclination angle of the TPET composite surface was set as $32^{\circ}$. The equilibrium temperature of the TPET composite surface with the change in solar irradiation and ambient temperature tested on July 22, 2014, was also calculated, and the results are shown in Figure 8. The simulation and experimental values demonstrated good agreement.

To further evaluate the solar heating and radiative cooling performance of the TPET surface, comparisons among the TPET surface and three other typical surfaces, namely, solar selective absorbing coating surface (conventional solar heating surface), polyvinyl-fluoride film coating with a sheet of evaporated aluminum (conventional radiative cooling surface), and ideal solar heating and radiative cooling composite surface (ideal composite surface), were performed according to spectral properties of each surface (Figures 4 and 6). Ge and Sun [10] provided related parameters.

A sample calculation was conducted under clear skies and a surface with no inclination angle, as well as in typical summer environmental conditions in Hefei, China (i.e., ambient temperature of $35^{\circ} \mathrm{C}$ during daytime and $30^{\circ} \mathrm{C}$ during nighttime and solar irradiation of $800 \mathrm{~W} / \mathrm{m}^{2}$ during daytime). The temperature of each surface was set $50^{\circ} \mathrm{C}$ during daytime and $25^{\circ} \mathrm{C}$ at nighttime. Comparisons of the characteristics of each surface and their heating and cooling performances are presented in Table 1.

Table 1 shows that the conventional solar heating surface has nearly no cooling effect, whereas the conventional radiative cooling surface has nearly no heating effect. The TPET composite surface and ideal composite surface have a solar heating and a radiative cooling effect. More specifically, in a solar heating model, the conventional solar heating surface had the highest heating efficiency. The TPET composite surface had a relative heat efficiency of $76.8 \%$ to the conventional solar heating surface compared with the ideal composite surface that had a relative heat efficiency of $80.6 \%$. 
TABLE 1: Comparisons of the characteristics of each surface and their heating and cooling performances under typical summer environmental conditions of Hefei, China.

\begin{tabular}{|c|c|c|c|c|c|c|c|c|}
\hline & Function & $\alpha_{0.2-3 \mu \mathrm{m}}$ & $\varepsilon_{8-13 \mu \mathrm{m}}$ & $\alpha / \varepsilon_{3-8 \mu \mathrm{m} \text { and } 13-25 \mu \mathrm{m}}$ & $\eta_{1} / \%$ & $\eta_{2} / \%$ & $\begin{array}{l}T_{r} \\
{ }^{\circ} \mathrm{C}\end{array}$ & $\begin{array}{c}q \\
\mathrm{~W} \cdot \mathrm{m}^{-2}\end{array}$ \\
\hline $\begin{array}{l}\text { Conventional solar } \\
\text { heating surface }\end{array}$ & Heating & 0.93 & 0.05 & 0.05 & 100 & $\approx 0$ & 29.2 & $\approx 0$ \\
\hline $\begin{array}{l}\text { Conventional } \\
\text { radiative cooling } \\
\text { surface }\end{array}$ & Cooling & 0.12 & 0.74 & 0.32 & $\approx 0$ & 100 & 10.8 & 36.1 \\
\hline $\begin{array}{l}\text { TPET composite } \\
\text { surface }\end{array}$ & $\begin{array}{l}\text { Heating and } \\
\text { cooling }\end{array}$ & 0.92 & 0.80 & 0.55 & 76.8 & 75.0 & 15.6 & 35.6 \\
\hline $\begin{array}{l}\text { Ideal composite } \\
\text { surface }\end{array}$ & $\begin{array}{l}\text { Heating and } \\
\text { cooling }\end{array}$ & 1.00 & 1.00 & 0 & 80.6 & 166 & -1.89 & 47.2 \\
\hline
\end{tabular}

${ }^{*} T_{r}$ and $q$ are equilibrium temperature and radiative cooling power of each surface, respectively.

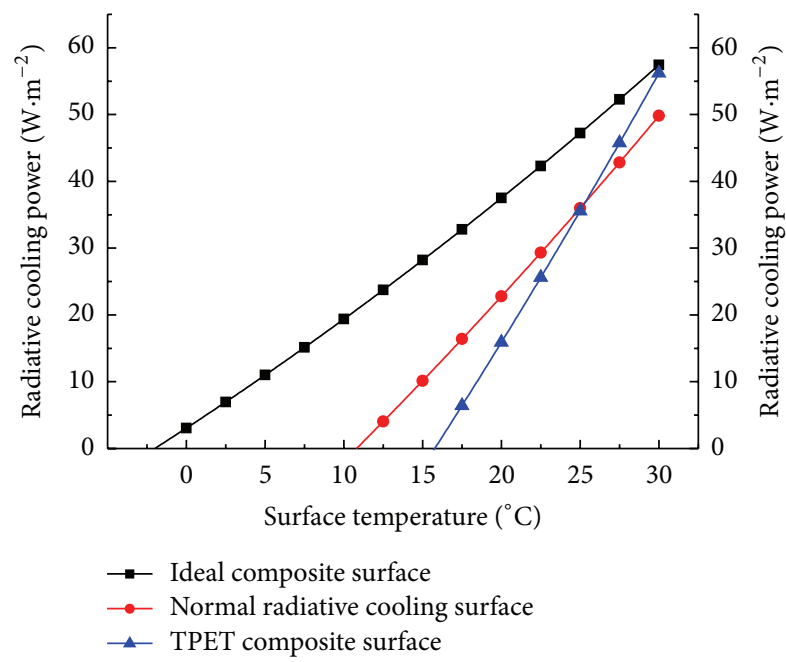

FIGURE 9: Change in radiative cooling power of the three kinds of radiative cooling surfaces with surface temperatures at ambient temperature of $30^{\circ} \mathrm{C}$.

In radiative cooling mode, the TPET composite surface had a cooling power of $36.1 \mathrm{~W} / \mathrm{m}^{2}$ at a surface temperature of $25^{\circ} \mathrm{C}$ and an equilibrium temperature of $15.6^{\circ} \mathrm{C}$. Under the same conditions, the conventional radiative cooling surface and the ideal composite surface had cooling powers of 35.6 and $47.2 \mathrm{~W} / \mathrm{m}^{2}$, as well as equilibrium temperatures of 10.8 and $-1.9^{\circ} \mathrm{C}$, respectively. Moreover, the TPET composite surface had a relative temperature difference of $75.0 \%$ relative to the conventional radiative cooling surface compared with the ideal composite surface that had a relative heat efficiency of $166 \%$.

In addition, the change in the radiative cooling power of the three kinds of radiative cooling surfaces with surface temperatures at ambient temperature of $30^{\circ} \mathrm{C}$ was examined and then illustrated in Figure 9. The radiative cooling power returned to zero when each surface reached its equilibrium temperature. The linear increase with the rise of surface temperatures was also presented. Moreover, the ideal composite surface always had the highest cooling powers when the surface temperature was lower than the ambient temperature.
When the surface temperature was higher than $25.2^{\circ} \mathrm{C}$, the cooling power of the TPET composite surface was greater than that of the conventional radiative cooling surface. Although the surface temperature was lower than $25.2^{\circ} \mathrm{C}$, the situation was reversed.

\section{Conclusion}

A spectral selectivity composite surface for both solar heating and radiative cooling was proposed in this paper based on properties and limitations of solar collectors and nocturnal radiators. This kind of spectral selectivity composite surface has high spectral absorptivity (emissivity) in solar radiation band (i.e., $0.2 \sim 3 \mu \mathrm{m}$ ) and atmospheric window band (i.e., 8 $13 \mu \mathrm{m}$ ) and has low spectral absorptivity (emissivity) in other bands. A type of composite surface used to fit in the spectral properties was trial-manufactured and designated as TPET composite surface. In this study, the spectral properties of the TPET composite surface were tested. Numerical analysis was performed to assess the solar heating and radiative cooling performance of the TPET surface by comparing it with three other typical surfaces. Experimental measurements were also carried out. The results helped provide the following conclusions:

(1) The TPET surface had high spectral absorptivity (emissivity) in solar radiation and atmospheric window band, indicating that it could act as a solar collector during the day and a nocturnal radiator during the night. In other bands aside from the solar radiation and atmospheric window wavelengths, it exhibited relatively low spectral absorptivity (emissivity), allowing it to decrease radiant heating loss during daytime and radiant cooling loss during nighttime.

(2) In the solar heating model, the heating efficiency of the TPET surface was lower than that of the conventional solar heating surface due to difference of spectral emissivity in the atmospheric window wavelength. However, the TPET surface continued to demonstrate considerable heating efficiency.

(3) In the radiative cooling model, the cooling power between the TPET surface and the conventional 
radiative cooling surface did not exhibit a large difference. The temperature of the radiation surface was a key factor to the cooling power. The higher the temperature of the radiation surface was, the larger the cooling power obtained.

(4) The sky condition and inclination angle of the surface were two other important cooling effect factors. The largest cooling power would appear when the sky was clear and the inclination angle was $0^{\circ}$. Throughout our tests, the temperature of the TPET surface was about $13^{\circ} \mathrm{C}$ lower than that of the ambient temperature under the most favorable condition for radiative cooling.

A system based on this kind of surface can not only address the shortcomings of conventional solar collectors and conventional nocturnal radiators but also shorten the initial payback period. Lowering further the spectral absorptivity (emissivity) of the TPET composite in bands aside from the solar radiation and atmospheric window wavelength or finding any other material whose spectral properties are closer to the ideal surface for both solar heating and radiative cooling will be emphasized in future research.

\section{Conflict of Interests}

The authors declare that there is no conflict of interests regarding the publication of this paper.

\section{Acknowledgments}

The study was sponsored by the (1) National Science Foundation of China (NSFC 51476159, NSFC 51178442) and the (2) Fundamental Research Funds for the Central Universities.

\section{References}

[1] D. Michell and K. L. Biggs, "Radiation cooling of buildings at night," Applied Energy, vol. 5, no. 4, pp. 263-275, 1979.

[2] S. Catalanotti, V. Cuomo, G. Piro, D. Ruggi, V. Silvestrini, and G. Troise, "The radiative cooling of selective surfaces," Solar Energy, vol. 17, no. 2, pp. 83-89, 1975.

[3] Y. Etzion and E. Erell, "Thermal storage mass in radiative cooling systems," Building and Environment, vol. 26, no. 4, pp. 389-394, 1991.

[4] E. Erell and Y. Etzion, "Analysis and experimental verification of an improved cooling radiator," Renewable Energy, vol. 16, no. 1-4, pp. 700-703, 1999.

[5] G. B. Smith, "Amplified radiative cooling via optimised combinations of aperture geometry and spectral emittance profiles of surfaces and the atmosphere," Solar Energy Materials \& Solar Cells, vol. 93, no. 9, pp. 1696-1701, 2009.

[6] S. Zhang and J. Niu, "Cooling performance of nocturnal radiative cooling combined with microencapsulated phase change material (MPCM) slurry storage," Energy and Buildings, vol. 54, pp. 122-130, 2012.

[7] H. S. Bagiorgas and G. Mihalakakou, "Experimental and theoretical investigation of a nocturnal radiator for space cooling," Renewable Energy, vol. 33, no. 6, pp. 1220-1227, 2008.
[8] M. Farmahini-Farahani and G. Heidarinejad, "Increasing effectiveness of evaporative cooling by pre-cooling using nocturnally stored water," Applied Thermal Engineering, vol. 38, pp. 117-123, 2012.

[9] M. G. Meir, J. B. Rekstad, and O. M. LØvvik, "A study of a polymer-based radiative cooling system," Solar Energy, vol. 73, no. 6, pp. 403-417, 2002.

[10] X. S. Ge and X. Sun, “The effect of radiator's spectrally selective nature to radiative cooling," Acta Energiae Solaris Sinica, vol. 3, no. 2, pp. 128-136, 1982.

[11] J. Li and Q. Jiang, "Experiments on radiative cooling," Acta Energiae Solaris Sinica, vol. 21, no. 3, pp. 243-247, 2000.

[12] C. G. Granqvist and A. Hjortsberg, "Radiative cooling to low temperatures: general considerations and application to selectively emitting SiO films," Journal of Applied Physics, vol. 52, no. 6, pp. 4205-4220, 1981.

[13] P. Berdahl, "Radiative cooling with $\mathrm{MgO}$ and/or LiF layers," Applied Optics, vol. 23, no. 3, pp. 370-372, 1984.

[14] Z. Rui and R. Zuo, "Experimental study on air-conditioning plant with space radiative cooling," Journal of Refrigeration, vol. 31, no. 4, pp. 57-62, 2010.

[15] T. S. Saitoh and T. Fujino, "Advanced energy-efficient house (HARBEMAN house) with solar thermal, photovoltaic, and sky radiation energies (Experimental results)," Solar energy, vol. 70, no. 1, pp. 63-77, 2001.

[16] S. N. Bathgate and S. G. Bosi, "A robust convection cover material for selective radiative cooling applications," Solar Energy Materials \& Solar Cells, vol. 95, no. 10, pp. 2778-2785, 2011.

[17] A. R. Gentle, K. L. Dybdal, and G. B. Smith, "Polymeric mesh for durable infra-red transparent convection shields: applications in cool roofs and sky cooling," Solar Energy Materials \& Solar Cells, vol. 115, pp. 79-85, 2013.

[18] M. Matsuta, S. Terada, and H. Ito, "Solar heating and radiative cooling using a solar collector-sky radiator with a spectrally selective surface," Solar Energy, vol. 39, no. 3, pp. 183-186, 1987.

[19] M. Mostrel and B. Givoni, "Windscreen in radiant cooling," Passive Solar Journal, vol. 1, no. 4, pp. 229-238, 1982. 

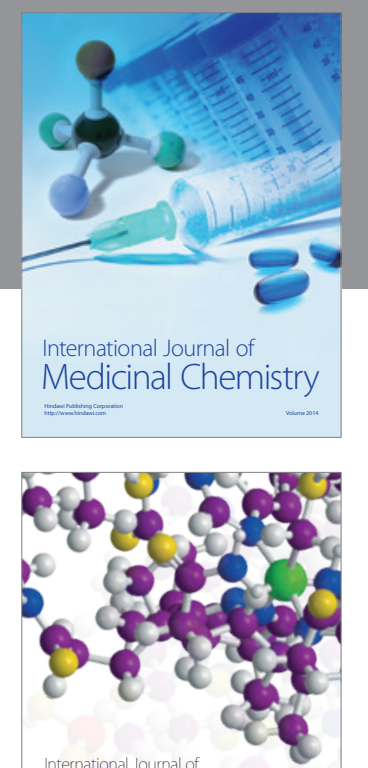

\section{Carbohydrate} Chemistry

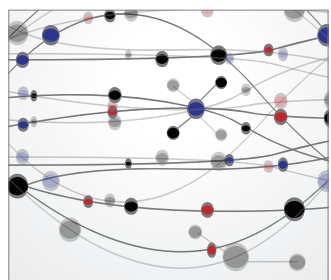

The Scientific World Journal
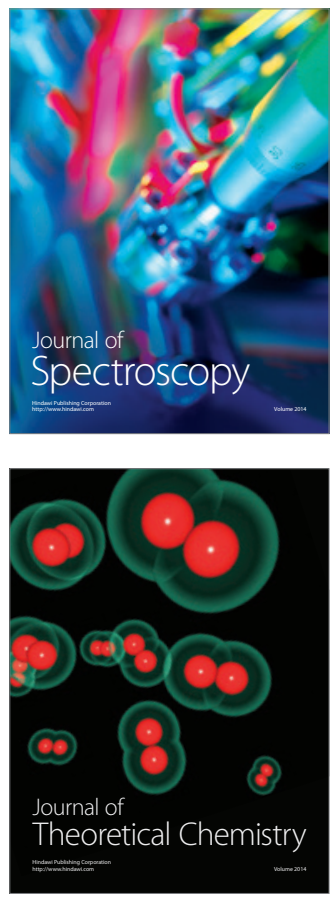
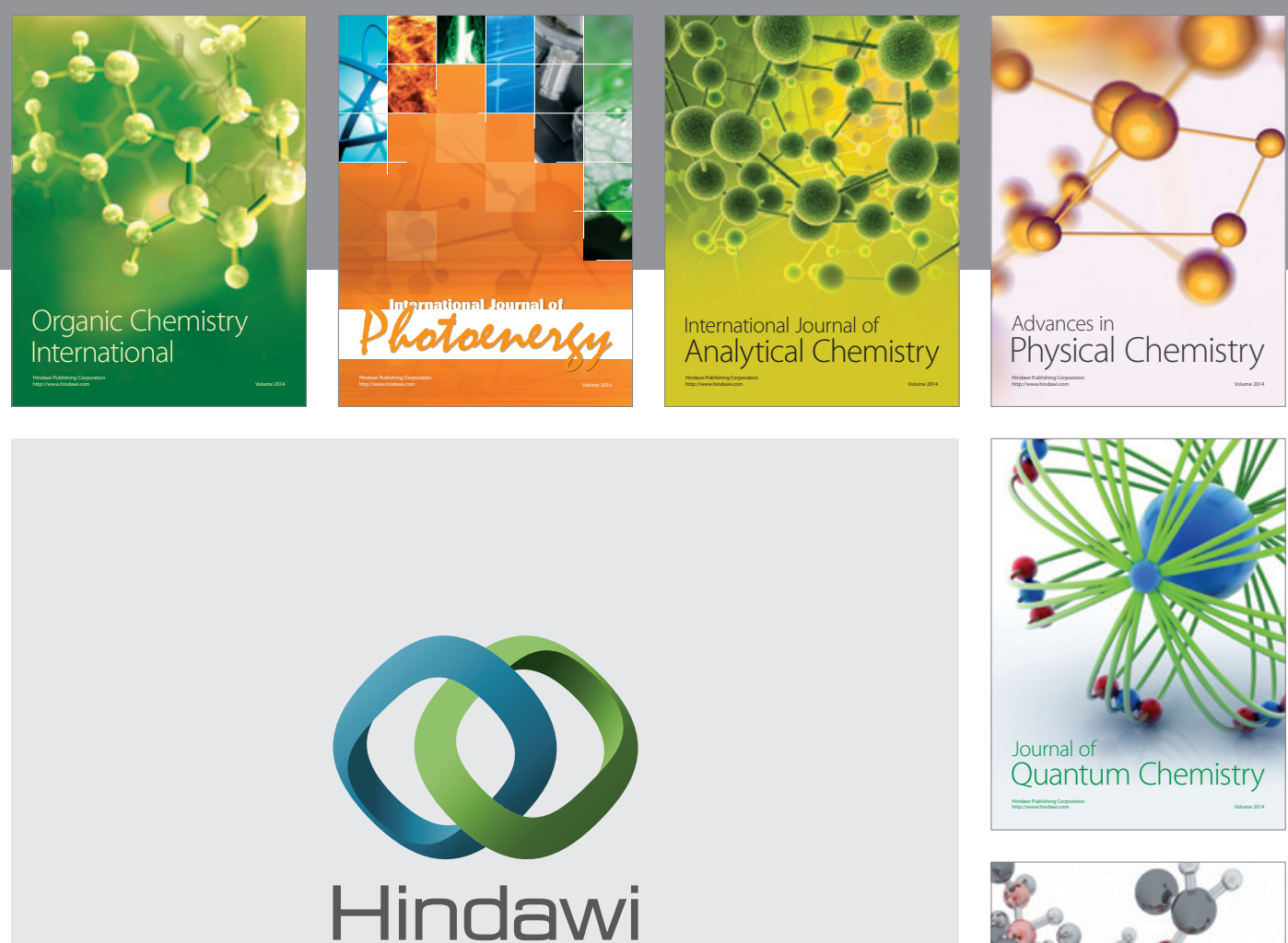

Submit your manuscripts at

http://www.hindawi.com

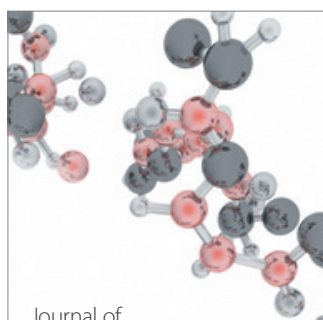

Analytical Methods

in Chemistry

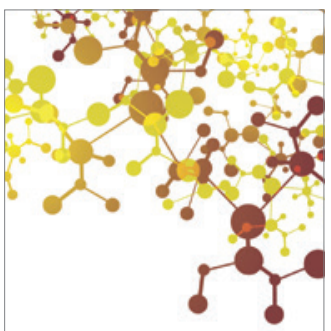

Journal of

Applied Chemistry

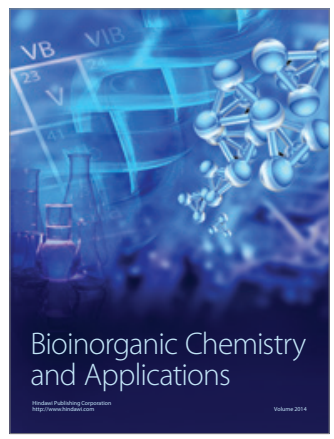

Inorganic Chemistry
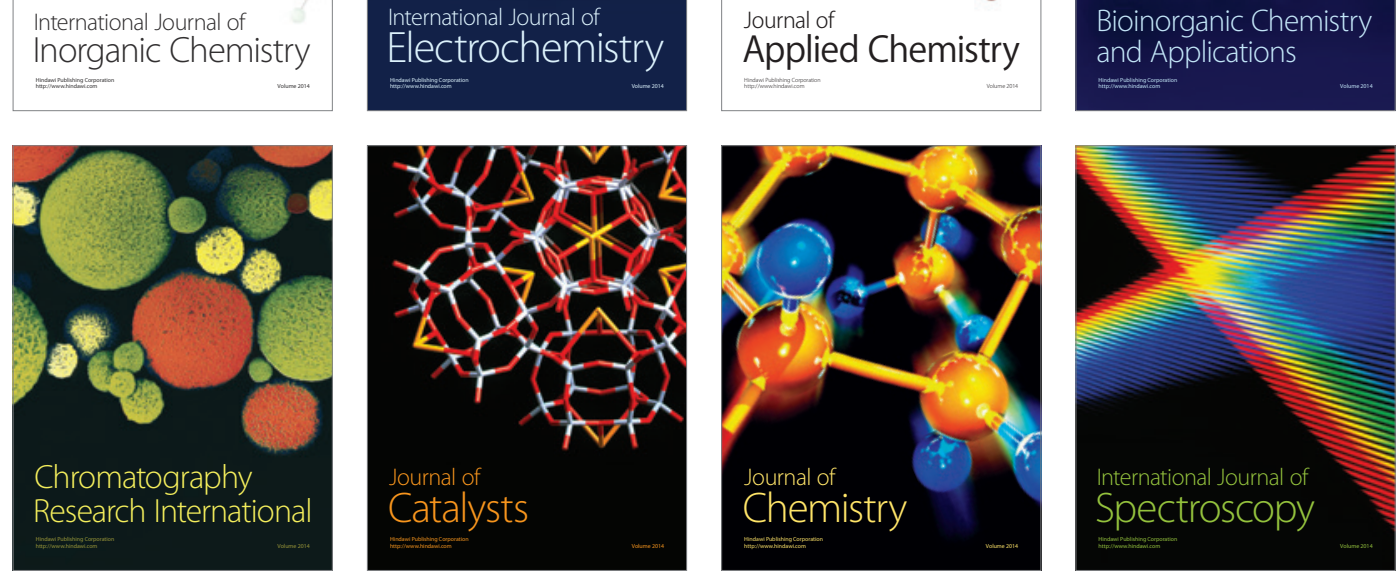